(2) Open Access Full Text Article

\title{
Design, synthesis, and antifungal activities of novel triazole derivatives containing the benzyl group
}

This article was published in the following Dove Press journal:

Drug Design, Development and Therapy

II March 2015

Number of times this article has been viewed

\author{
Kehan $\mathrm{Xu}^{1, *}$ \\ Lei Huang ${ }^{1, *}$ \\ Zheng $\mathrm{Xu}^{2}$ \\ Yanwei Wang ${ }^{1,3}$ \\ Guojing Bai' \\ Qiuye $\mathrm{Wu}^{\prime}$ \\ Xiaoyan Wang' \\ Shichong Yu' \\ Yuanying Jiang' \\ 'School of Pharmacy, Second Military \\ Medical University, Shanghai, ${ }^{2}$ Shanghai \\ Changzheng Hospital, Second Military \\ Medical University, Shanghai, ${ }^{3}$ Number \\ 422 Hospital of PLA, Zhanjiang, \\ People's Republic of China \\ *These authors contributed equally \\ to this work
}

\begin{abstract}
In previous studies undertaken by our group, a series of 1-(1H-1,2,4-triazole-1-yl)-2(2,4-difluorophenyl)-3-substituted-2-propanols (1a-r), which were analogs of fluconazole, was designed and synthesized by click chemistry. In the study reported here, the in vitro antifungal activities of all the target compounds were evaluated against eight human pathogenic fungi. Compounds 1a, 1q, and 1r showed the more antifungal activity than the others.
\end{abstract}

Keywords: triazole, synthesis, antifungal activity, CYP51

\section{Introduction}

In the past three decades, deep fungal infections have sharply escalated due to the employment of clinical antitumor drugs and immunosuppressants; the widespread application of broad-spectrum antibiotics, cancer chemotherapy, radiotherapy, peritoneal dialysis, organ transplantation; and immune deficiency disorders, especially AIDS. ${ }^{1,2}$ Currently, aspergillosis, cryptococcosis, and candidiasis are three major clinical fungal infections in immunocompromised individuals. ${ }^{3,4}$ Azole nitrogen compounds have been progressively getting people's attention, mainly because of their superiority in antifungal therapy but also for their contribution in the treatment of various microbes. ${ }^{5}$ Azoles (fluconazole, itraconazole, voriconazole, and posaconazole, Figure 1) are one very significant class of compounds for treating deep fungal infections in the clinical context. ${ }^{6}$ One of the principal problems in the treatment of Candida albicans infections is the spread of antifungal drug resistance, mainly in patients chronically subjected to antimycotic therapy such as HIV-infected people. ${ }^{7,8}$

More recently, there has been a development in the number of antifungal drugs available. Five major classes of antifungal compounds are currently in clinical use: polyenes, azole derivatives, allylamines, thiocarbamates, and fluoropyrimidines. ${ }^{9-12}$ In spite of this growing list of antifungal agents in the process of being studied, treatment of fungal diseases remains unsatisfactory. The limitations of current antifungal drugs, increased incidence of systemic fungal infections, and rapid development of drug resistance have emphasized the need for the discovery of new antifungal agents with a new mode of action and fewer side effects. ${ }^{4,10,13-15}$

In particular, azole drugs are very important antifungal agents widely used in the clinical context. ${ }^{16}$ Azoles exert antifungal activity through the inhibition of cytochrome P450 14 $\alpha$-demethylase (CYP51), which is crucial in the process of biosynthesis of ergosterol. The CYP51 enzyme contains an iron protoporphyrin unit located in its active site, which catalyzes the oxidative removal of the $14 \alpha$-methyl group of lanosterol by typical monooxygenase activity. ${ }^{17}$ Azole antifungal agents bind to the iron of the porphyrin and cause blockade of the fungal ergosterol biosynthesis pathway 
<smiles>OC(Cn1cncn1)(Cn1cncn1)c1ccc(F)cc1F</smiles>

Fluconazole<smiles>CC(c1ncncc1F)C(O)(Cn1cncn1)c1ccc(F)cc1F</smiles>

Voriconazole<smiles>CCC(C)n1ncn(-c2ccc(N3CCN(c4ccc(Oc5ccc([C@]6(Cn7cncn7)OC[C@H](C)O6)cc5)cc4)CC3)cc2)c1=O</smiles>

Itraconazole

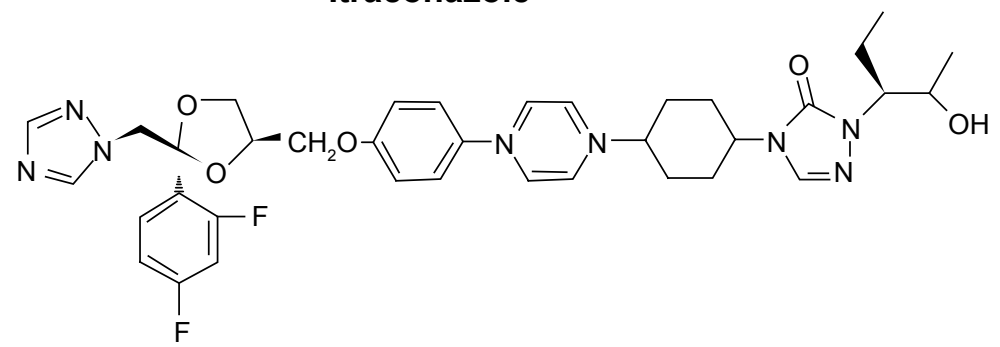

Posaconazole

Figure I Triazole antifungal agents used in clinical therapy.

by preventing the access of the natural substrate lanosterol to the active site of the enzyme. ${ }^{18}$

In previous research by our group, ${ }^{19-27}$ numerous studies on the structure-activity relationships (SAR) of antifungal azoles were undertaken, and these studies led to new compounds endowed with better biological and pharmacological properties. These studies indicated that the triazole ring, the difluorophenyl group, and the hydroxyl group are the pharmacophores of antifungal agents. We focused our attention on installing various substituted benzyl groups of the side chain by click chemistry.

According to the results of these studies, we designed a series of 1-(1H-1,2,4-triazole-1-yl)-2-(2,4-difluorophenyl)3-substituted-2-propanols (1a-r, Figure 2) containing a triazole ring, a difluorophenyl group, a hydroxyl group, and a side chain containing a piperazine group. In our design, we systematically altered the structure of fluconazole as a

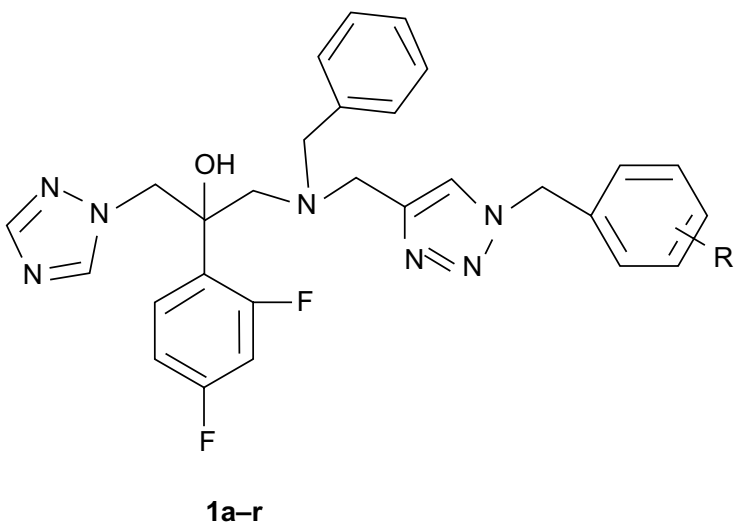

Figure 2 Generic structure of the designed fluconazole analogs. platform and tried to insert a 1,2,3-triazole group into the side chain.

\section{Chemistry}

The general synthetic methodology for the preparation of the title compounds (1a-r) is outlined in Figure 3. Compound 3 was synthesized by ring-open reaction of oxirane 2 with benzylamine. Then, compound 3 was transformed into compound 4 by reacting with propargyl bromide in the presence of $\mathrm{KI}$ and $\mathrm{K}_{2} \mathrm{CO}_{3}$ in acetonitrile. The target compounds were obtained by using click chemistry ${ }^{28}$ with various substituted benzyl azides.

\section{Pharmacology}

The in vitro antifungal activities of all the target compounds were evaluated against eight human pathogenic fungi C. albicans 14053, C. albicans 20352, Candida parapsilosis, Cryptococcus neoformans, Candida glabrata, Aspergillus fumigatus, Trichophyton rubrum, and Microsporum gypseumwhich are often encountered clinically, and were compared with itraconazole (ICZ), voriconazole (VCZ), and fluconazole (FCZ). All eight human pathogenic fungi were provided by Shanghai Changzheng Hospital; FCZ, ICZ, and VCZ, which served as the positive control, were obtained from their respective manufacturers.

The in vitro minimal inhibitory concentrations (MICs) of the compounds were determined by the micro-broth dilution method in 96-well micro test plates according to the methods defined by the National Committee for Clinical Laboratory Standards. ${ }^{29}$ The MIC80 was defined as the first well containing an approximate $80 \%$ reduction in growth compared with 

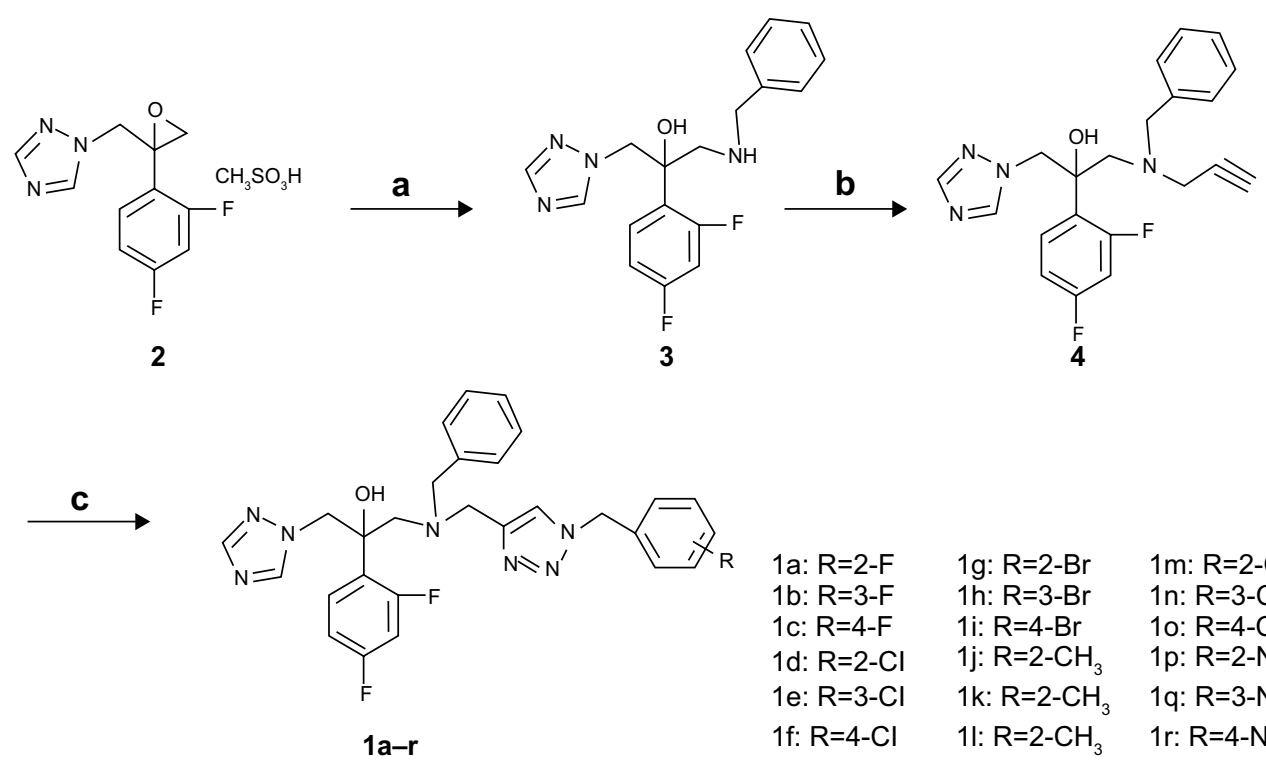

1a: $R=2-F$
1b: $R=3-F$
1c: $R=4-F$
1d: $R=2-C l$
1e: $R=3-C l$
1f: $R=4-C l$

$\begin{array}{ll}\text { 1g: } R=2-B r & 1 \mathrm{~m}: \mathrm{R}=2-\mathrm{CN} \\ \text { 1h: } R=3-\mathrm{Br} & 1 \mathrm{n}: \mathrm{R}=3-\mathrm{CN} \\ \text { 1i: } \mathrm{R}=4-\mathrm{Br} & \text { 1o: } \mathrm{R}=4-\mathrm{CN} \\ \text { 1j: } \mathrm{R}=2-\mathrm{CH}_{3} & \text { 1p: } \mathrm{R}=2-\mathrm{NO}_{2} \\ \text { 1k: } \mathrm{R}=2-\mathrm{CH}_{3} & \text { 1q: } \mathrm{R}=3-\mathrm{NO}_{2} \\ \text { 1l: } \mathrm{R}=2-\mathrm{CH}_{3} & \text { 1r: } \mathrm{R}=4-\mathrm{NO}_{2}\end{array}$

Figure 3 Synthesis of the target compounds Ia-r.

Notes: Conditions: (a) $\mathrm{Et}_{3} \mathrm{~N}$, benzylamine, $\mathrm{EtOH} \mathrm{Et}_{3} \mathrm{~N}$, reflux, 5 hours, $72 \%$; (b) propargyl bromide, $\mathrm{KI}_{2} \mathrm{~K}_{2} \mathrm{CO}_{3}, \mathrm{CH}_{3} \mathrm{CN}, \mathrm{rt}, 5-6$ hours, $81 \%$; (c) $\mathrm{NaN}$, substituted benzyl bromide, dimethyl sulfoxide, $\mathrm{CuSO}_{4} \cdot 5 \mathrm{H}_{2} \mathrm{O}$, sodium ascorbate, $\mathrm{rt}, 12$ hours, $60 \%-70 \%$.

growth in the drug-free well. For assays, the title compounds to be tested were dissolved in dimethyl sulfoxide (DMSO), serially diluted in growth medium, inoculated, and incubated at $35^{\circ} \mathrm{C}$. The growth MIC was determined at 24 hours for C. albicans and at 72 hours for C. neoformans.

\section{Materials and methods}

Melting points (MPs) were measured on a YRT-3 Melting Point Tester (Tianda Tianfa Technology Co., LTD, Tianjin, People's Republic of China) and are presented uncorrected. Proton nuclear magnetic resonance $\left({ }^{1} \mathrm{H}\right.$ NMR) spectra are recorded in $\mathrm{CDCl}_{3}$, unless otherwise indicated, with an Avance II 300 spectrometer (Bruker Corporation, Billerica, MA, USA), using tetramethlysilane as the internal standard. Electrospray ionization-mass spectrometry (ESI-MS) spectra were obtained using an API 3000 liquid chromatography-mass spectrometry spectrometer (Thermo Fisher Scientific, Waltham, MA, USA). Thin-layer chromatography analysis was carried out on GF254 silica gel plates (Qingdao Haiyang Chemical Co Ltd, Qingdao, People's Republic of China). Column chromatography was performed with silica gel $60 \mathrm{G}$ (Qingdao Haiyang Chemical Co Ltd). The solvents and reagents were used as received or dried prior to use, as needed.

\section{Compound 3: I-( $\mid \mathrm{H}-\mathrm{I}, 2,4$-triazol- $|-\mathrm{y}|)-$ 2-(2,4-difluorophenyl)-3-(benzylamino)- 2-propanol}

To a stirred mixture of 1-[2-(2,4-difluorophenyl)-2,3epoxypropyl]-1H-1,2,4-triazole methanesulfonate (2) (16.5 $\mathrm{g}, 0.05 \mathrm{~mol}), \mathrm{C}_{2} \mathrm{H}_{5} \mathrm{OH}(200 \mathrm{~mL})$ and $\mathrm{N}\left(\mathrm{C}_{2} \mathrm{H}_{5}\right)_{3}(30 \mathrm{~mL})$, benzylamine $(6.42 \mathrm{~g}, 0.06 \mathrm{~mol})$ were added and heated at $70^{\circ} \mathrm{C}-80^{\circ} \mathrm{C}$ for 5 hours. The reaction was monitored by thin layer chromatography. After filtration, the filtrate was evaporated under reduced pressure. Water was added to the residue, which was then extracted with ethyl acetate. The extract was washed with saturated $\mathrm{NaCl}$ solution, dried over anhydrous $\mathrm{Na}_{2} \mathrm{SO}_{4}$, and evaporated. The residue was separated and purified readily by chromatography on silica gel to afford Compound 3 (12.4 g, 72\% yield).

Compound 4: I-(IH-I,2,4-triazol-I-y|)2-(2,4-difluorophenyl)-3-( $N$-benzyl- $N$ propargyl amino)-2-propanol

To a stirred mixture, Compound 3 (3.44 g, $0.01 \mathrm{~mol})$, propargyl bromide (2.36 g, $0.02 \mathrm{~mol})$, KI (166 mg, 0.001 $\mathrm{mol}), \mathrm{K}_{2} \mathrm{CO}_{3}(3.45 \mathrm{~g}, 0.025 \mathrm{~mol})$, and $\mathrm{CH}_{3} \mathrm{CN}(50 \mathrm{~mL})$ was stirred at room temperature for 6 hours. The reaction was monitored by thin layer chromatography. When the reaction was completed, the solid was filtrated, washed with $\mathrm{CH}_{3} \mathrm{CN}$, then the filtrate was concentrated in a vacuum. Column chromatography of the residue afforded Compound 4 as an oil (3.09 $\mathrm{g}, 81 \%$ yield).

\section{General procedure for the preparation of Compounds Ia-r}

A mixture of $\mathrm{NaN}_{3}$ (100 mg, $\left.1.4 \mathrm{mmol}\right)$, 2-fluorobenzyl bromide (200 mg, $1.2 \mathrm{mmol}$ ), and DMSO (15 mL) was stirred at room temperature for 6 hours. Then, to this was added Compound 4 (229 mg, $0.6 \mathrm{mmol}$ ), sodium ascorbate (20 mg), $\mathrm{CuSO}_{4} \cdot 5 \mathrm{H}_{2} \mathrm{O}(25 \mathrm{mg})$, and $\mathrm{H}_{2} \mathrm{O}(1 \mathrm{~mL})$. The mixture 


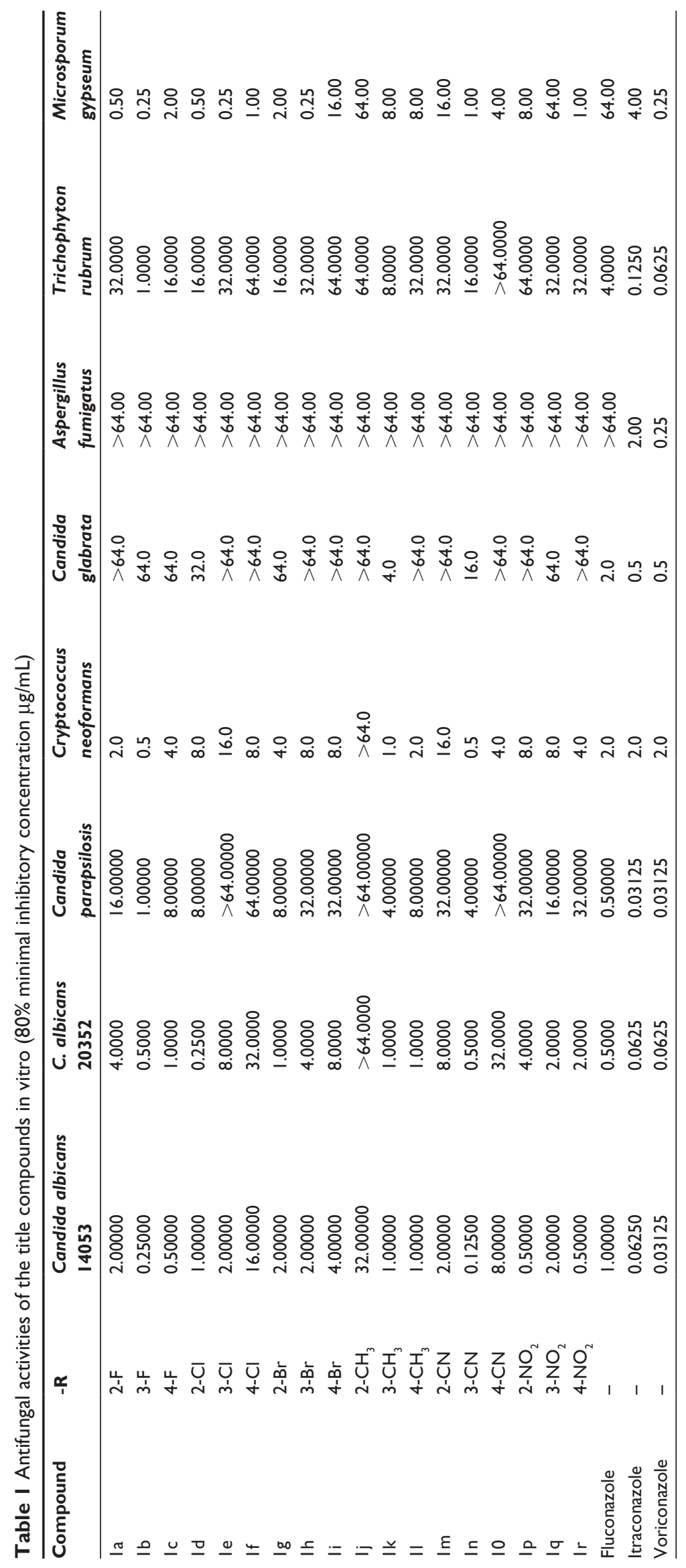


was stirred at room temperature for 2 hours, then $\mathrm{NH}_{3} \cdot \mathrm{H}_{2} \mathrm{O}$ was added carefully, then extracted with ethyl acetate. The organic layer was acidified with dilute hydrochloric acid, then the $\mathrm{pH}$ of the aqueous layer was adjusted to about 7.0 by saturation with sodium bicarbonate, then the solution was extracted with ethyl acetate, washed with water, $\mathrm{NaHCO}_{3}$ and $\mathrm{NaCl}$ solutions, dried with $\mathrm{Na}_{2} \mathrm{SO}_{4}$, and concentrated in a vacuum to afford Compound 1a (212 mg, 69\% yield; MP, $92.0^{\circ} \mathrm{C}-94.0^{\circ} \mathrm{C} ;{ }^{1} \mathrm{H}$ NMR $\left[300 \mathrm{MHz}, \mathrm{CDCl}_{3}\right] \delta: 8.06[1 \mathrm{H}$, $\mathrm{s}$, triazole-H], $7.72[1 \mathrm{H}, \mathrm{s}$, triazole- $\mathrm{H}], 7.63-7.56[1 \mathrm{H}, \mathrm{m}$, $\mathrm{Ar}$, 7.39-7.12 [8H, m, Ar-H, triazole-H], 6.83-6.69 [2H, $\mathrm{m}, \mathrm{Ar}-\mathrm{H}], 5.60$ [2H, s, Ar- $\left.\mathrm{CH}_{2}-\right], 4.50-4.36$ [2H, m, $\left.\mathrm{CH}_{2}\right]$, 3.72-3.38 [4H, m, - $\mathrm{CH}_{2}-\mathrm{N}-\mathrm{CH}_{2}-$ ], 3.28-2.90 [2H, m, $\mathrm{CH}_{2}$ ], ${ }^{13} \mathrm{C}$ NMR $\left[75 \mathrm{MHz}, \mathrm{CDCl}_{3}\right]$ $\delta: 163.2,159.0,151.1,130.2$, 130.0, 129.9, 129.9, 129.8, 129.8, 129.8, 129.7, 129.6, 129.6, $129.5,129.5,129.4,129.3,128.5,128.0,116.2,111.8,104.4$, 73.0, 59.7, 57.5, 56.0, 35.6, 49.2; ESI-MS, m/z calculated for $\mathrm{C} 28 \mathrm{H} 26 \mathrm{~F} 3 \mathrm{~N} 7 \mathrm{O}, 533.2$, found $[\mathrm{M}+\mathrm{H}]+534.5$ ). (The characterization of Compounds b-r is presented in the "Supplementary materials" section).

\section{Results}

The in vitro antifungal activities are summarized in Table 1, along with the MIC values (in $\mu \mathrm{g} / \mathrm{mL}$ ) against different pathogenic fungi, in comparison with ICZ, VCZ, and FCZ. The results of the study of the antifungal activities in vitro show that all 18 target compounds (1a-r) were active against nearly all fungi tested to some extent, except against $A$. fumigatus and C. glabrata. The MIC80 value of Compounds $1 \mathrm{a}$ and $1 \mathrm{~h}$ was four times lower than that of FCZ against C. albicans 14053 in vitro (with an MIC80 value of $0.25 \mu \mathrm{g} / \mathrm{mL}$ ). The MIC80 value of Compounds 1a, 1q, and 1r was 256 times lower than that of FCZ against M. gypseum in vitro, and the same as VCZ against $M$. gypseum in vitro (with an MIC80 value of $0.25 \mu \mathrm{g} / \mathrm{mL}$ ). The MIC80 value of most target compounds against $C$. neoformans was the same as that of the control drugs. However, most of the target compounds' antifungal activities were not as good as those of ICZ and VCZ.

\section{Conclusion}

A series of triazoles was successfully synthesized and characterized by ESI-MS and nuclear magnetic resonance spectroscopic analysis. In vitro antifungal activity assay indicated that most of the compounds showed antifungal activities against both systemic pathogenic fungi. The MIC80 value of the compounds in which halogen was substituted to position three against $M$. gypseum was better than that of the other compounds. Several compounds showed high in vitro antifungal activity that was broad spectrum, which will be valuable to future investigations.

\section{Acknowledgments}

This work was supported by the National Key Basic Research Program of China (no 2013CB531602), the National Natural Science Foundation of China (no 81330083), the Creativity and Innovation Training Program of Second Military Medical University, and the Pharmaceutical Education Research Project of the Chinese Association of Higher Medical Education Professional Committee (grant no ZD201220).

Electronic supplementary information available: proton nuclear magnetic resonance and electrospray ionization-mass spectrometry spectral data of Compounds $1 \mathrm{~b}-\mathrm{r}$.

\section{Disclosure}

The authors report no conflicts of interest in this work.

\section{References}

1. Wingard JR, Leather H. A new era of antifungal therapy. Biol Blood Marrow Transplant. 2004;10(2):73-90.

2. Fridkin SK, Jarvis WR. Epidemiology of nosocomial fungal infections. Clin Microbiol Rev. 1996;9(4):499-511.

3. Sheng CQ, Zhang WN, Ji HT, et al. Design, synthesis and antifungal activity of novel triazole derivatives. Chin Chem Lett. 2004; 15(4):404-407.

4. Sheng C, Zhang W, Ji H, et al. Structure-based optimization of azole antifungal agents by CoMFA, CoMSIA, and molecular docking. J Med Chem. 2006;49(8):2512-2525.

5. Gadhave PP, Dighe NS, Pattan SR, Deotarse P, Musmade DS, Shete RV. Current biological and synthetic profile of triazoles: a review. Ann Biol Res. 2010;1(1):82-89.

6. Groll AH, Lumb J. New developments in invasive fungal disease. Future Microbiol. 2012;7(2):179-184.

7. Wildfeuer A, Seidl HP, Paule I, Haberreiter A. In vitro evaluation of voriconazole against clinical isolates of yeasts, moulds and dermatophytes in comparison with itraconazole, ketoconazole, amphotericin B and griseofulvin. Mycoses. 1998;41(7-8):309-319.

8. Georgopapadakou NH. Antifungals: mechanism of action and resistance, established and novel drugs. Curr Opin Microbiol. 1998;1(5): $547-557$.

9. Kontoyiannis DP, Mantadakis E, Samonis G. Systemic mycoses in the immunocompromised host: an update in antifungal therapy. $J$ Hosp Infect. 2003;53(4):243-258.

10. Ghannoum MA, Rice LB. Antifungal agents: mode of action, mechanisms of resistance, and correlation of these mechanisms with bacterial resistance. Clin Microbiol Rev. 1999;12(4):501-517.

11. White TC, Marr KA, Bowden RA. Clinical, cellular, and molecular factors that contribute to antifungal drug resistance. Clin Microbiol Rev. 1998;11(2):382-402.

12. Wong-Beringer A, Kriengkauykiat J. Systemic antifungal therapy: new options, new challenges. Pharmacotherapy. 2003;23(11):1441-1462.

13. Lepesheva GI, Hargrove TY, Ott RD, Nes WD, Waterman MR. Biodiversity of CYP51 in trypanosomes. Biochem Soc Trans. 2006;34(Pt 6): 1161-1164.

14. Pfaller MA, Messer SA, Hollis RJ, Jones RN. In vitro activities of posaconazole (Sch 56592) compared with those of itraconazole and fluconazole against 3,685 clinical isolates of Candida spp and Cryptococcus neoformans. Antimicrob Agents Chemother. 2001; 45(10):2862-2864. 
15. Sheehan DJ, Hitchcock CA, Sibley CM. Current and emerging azole antifungal agents. Clin Microbiol Rev. 1999;12(1):40-79.

16. Lewis RE. Current concepts in antifungal pharmacology. Mayo Clin Proc. 2011;86(8):805-817.

17. Di Santo R, Tafi A, Costi R, et al. Antifungal agents. 11. N-substituted derivatives of 1-[(aryl)(4-aryl-1H-pyrrol-3-yl)methyl]-1H-imidazole: synthesis, anti-Candida activity, and QSAR studies. J Med Chem. 2005;48(16):5140-5153.

18. Odds FC, Brown AJ, Gow NA. Antifungal agents: mechanisms of action. Trends Microbiol. 2003;11(6):272-279.

19. Zou Y, Yu S, Li R, et al. Synthesis, antifungal activities and molecular docking studies of novel 2-(2,4-difluorophenyl)-2-hydroxy-3-(1H-1, 2,4-triazol-1-yl)propyl dithiocarbamates. Eur J Med Chem. 2014;74: 366-374.

20. Yu S, Chai X, Wang N, et al. Synthesis and antifungal activity of the novel triazole compounds. Med Chem Commun. 2013;4(4): 704-708.

21. Yu S, Wang L, Wang Y, et al. Molecular docking, design, synthesis and antifungal activity study of novel triazole derivatives containing the 1,2,3-triazole group. RSC Adv. 2013;3(32):13486-13490.

22. Yu S, Chai X, Hu H, et al. Synthesis and antifungal evaluation of novel triazole derivatives as inhibitors of cytochrome P450 14alphademethylase. Eur J Med Chem. 2010;45(10):4435-4445.
23. Wang Y, Xu K, Bai G, et al. Synthesis and antifungal activity of novel triazole compounds containing piperazine moiety. Molecules. 2014; 19(8):11333-11340.

24. Wang BG, Yu SC, Chai XY, Yan YZ, Hu HG, Wu QY. Design synthesis and biological evaluation of 3-substituted triazole derivatives. Chin Chem Lett. 2011;22(5):519-522.

25. Wang N, Chai X, Chen Y, et al. Synthesis, antifungal activity, and molecular docking studies of novel triazole derivatives. Med Chem. 2013; 9(3):384-388

26. Yu S, Chai X, Wang Y, et al. Triazole derivatives with improved in vitro antifungal activity over azole drugs. Drug Des Devel Ther. 2014; 8:383-390

27. Yu S, Wang Q, Zhang J, Wu Q, Guo Z. Synthesis and Evaluation of Protein Conjugates of GM3 Derivatives Carrying Modified Sialic Acids as Highly Immunogenic Cancer Vaccine Candidates. Medchemcomm. 2011;2(6):524-530.

28. Zhang XJ, Li HY, You LF, Tang Y, Hsung RP. Copper salt-catalyzed azide-[3+2] cycloadditions of ynamides and bis-ynamides. Adv Synth Catal. 2006;348(16-17):2437-2442.

29. National Committee for Clinical Laboratory Standards. Reference Method for Broth Dilution Antifungal Susceptibility Testing of Yeasts: Approved Standard. Document M27-A2. Wayne, PA: National Committee for Clinical Laboratory Standards; 2002. 


\section{Supplementary materials}

The title compounds $1 \mathrm{~b}-\mathrm{r}$ were characterized as follows.

Compound Ib: I-(IH-I,2,4-triazol- I-yl)2-(2,4-difluorophenyl)-3-\{N-benzyl- $N$ [( I-(3-fluorobenzyl)- IH-I,2,3-triazol-4-yl) methyl]amino\}-2-propanol

Melting point (MP): $94.1^{\circ} \mathrm{C}-96.0^{\circ} \mathrm{C}$; proton nuclear magnetic resonance $\left({ }^{1} \mathrm{H} \mathrm{NMR}\right)\left(300 \mathrm{MHz}, \mathrm{CDCl}_{3}\right) \delta: 8.13$ $(1 \mathrm{H}$, s, triazole-H), $7.77(1 \mathrm{H}, \mathrm{s}$, triazole- $\mathrm{H}), 7.64-7.55(1 \mathrm{H}$, $\mathrm{m}, \mathrm{Ar}), 7.37-7.12(8 \mathrm{H}, \mathrm{m}, \mathrm{Ar}-\mathrm{H}$, triazole-H), 6.82-6.74 (2H, $\mathrm{m}, \mathrm{Ar}-\mathrm{H}), 5.46\left(2 \mathrm{H}, \mathrm{s}, \mathrm{Ar}-\mathrm{CH}_{2}-\right), 4.51-4.36\left(2 \mathrm{H}, \mathrm{m}, \mathrm{CH}_{2}\right)$, 3.71-3.36 (4H, m, - $\left.\mathrm{CH}_{2}-\mathrm{N}-\mathrm{CH}_{2}-\right)$, 3.27-2.91 (2H, m, $\left.\mathrm{CH}_{2}\right)$; carbon-13 nuclear magnetic resonance $\left({ }^{13} \mathrm{C} \mathrm{NMR}\right)(75 \mathrm{MHz}$, $\left.\mathrm{CDCl}_{3}\right) \delta: 163.3,159.1,151.2,130.3,130.1,129.9,129.9$, $129.9,129.7,129.7,129.7,129.6,129.6,129.6,129.5,129.5$, 129.4, 128.6, 128.1, 116.3, 111.9, 104.5, 73.1, 59.8, 57.6, $56.1,35.7,49.3$; electrospray ionization-mass spectrometry (ESI-MS), $\mathrm{m} / \mathrm{z}$ calculated for $\mathrm{C}_{28} \mathrm{H}_{26} \mathrm{~F}_{3} \mathrm{~N}_{7} \mathrm{O}, 533.2$, found $[\mathrm{M}+\mathrm{H}]^{+}$534.3.

Compound I c: I-(|H-I,2,4-triazol- I-y|)2-(2,4-difluorophenyl)-3-\{N-benzyl- $N$ [( I-(4-fluorobenzyl)- IH-I,2,3-triazol-4-yl) methyl]amino\}-2-propanol (Ic) MP: $94.1^{\circ} \mathrm{C}-96.0^{\circ} \mathrm{C} ;{ }^{1} \mathrm{H}$ NMR $\left(300 \mathrm{MHz}, \mathrm{CDCl}_{3}\right)$ ): 8.11 $(1 \mathrm{H}, \mathrm{s}$, triazole-H), $7.72(1 \mathrm{H}, \mathrm{s}$, triazole-H), 7.69-7.57 $(1 \mathrm{H}$, $\mathrm{m}, \mathrm{Ar}), 7.29-7.06(8 \mathrm{H}, \mathrm{m}, \mathrm{Ar}-\mathrm{H}$, triazole-H), 6.84-6.69 $(2 \mathrm{H}$, $\mathrm{m}, \mathrm{Ar}-\mathrm{H}), 5.51\left(2 \mathrm{H}, \mathrm{s}, \mathrm{Ar}-\mathrm{CH}_{2}-\right), 4.60-4.36\left(2 \mathrm{H}, \mathrm{m}, \mathrm{CH}_{2}\right)$, 3.67-3.34 (4H, m, - $\mathrm{CH}_{2}-\mathrm{N}-\mathrm{CH}_{2}-$ ), 3.01-2.64 (2H, m, $\left.\mathrm{CH}_{2}\right)$; ${ }^{13} \mathrm{C}$ NMR (75 MHz, $\mathrm{CDCl}_{3}$ ) $\delta: 163.1,159.0,151.0,130.1$, 130.0, 129.9, 129.9, 129.8, 129.8, 129.7, 129.7, 129.7, 129.6, 129.6, 129.6, 129.5, 129.4, 128.7, 128.0, 116.1, 111.7, 104.2, 73.2, 59.7, 57.5, 56.0, 35.5, 49.2; ESI-MS, m/z calculated for $\mathrm{C}_{28} \mathrm{H}_{26} \mathrm{~F}_{3} \mathrm{~N}_{7} \mathrm{O}, 533.2$, found $[\mathrm{M}+\mathrm{H}]^{+}$534.4.

Compound Id: I-(IH-I,2,4-triazol- I-yl)2-(2,4-difluorophenyl)-3-\{N-benzyl- $N$ [( I-(2-chlorobenzyl)- IH-I,2,3-triazol-4yl)methyl]amino\}-2-propanol

MP: $114.6^{\circ} \mathrm{C}-116.0^{\circ} \mathrm{C} ;{ }^{1} \mathrm{H}$ NMR $\left(300 \mathrm{MHz}, \mathrm{CDCl}_{3}\right) \delta: 8.01$ $(1 \mathrm{H}, \mathrm{s}$, triazole-H), $7.71(1 \mathrm{H}, \mathrm{s}$, triazole- $\mathrm{H}), 7.64-7.55(1 \mathrm{H}$, m, Ar-H), 7.47-7.11 (8H, m, Ar-H, triazole-H), 6.80-6.68 $(2 \mathrm{H}, \mathrm{m}, \mathrm{Ar}-\mathrm{H}), 5.65$ (2H, s, Ar- $\left.\mathrm{CH}_{2}-\right), 4.50-4.36$ (2H, m, triazole-H), 3.68-3.39 (4H, m, $\left.-\mathrm{CH}_{2}-\mathrm{N}_{-} \mathrm{CH}_{2}-\right), 3.27-2.83$ $\left(2 \mathrm{H}, \mathrm{m}, \mathrm{CH}_{2}\right) ;{ }^{13} \mathrm{C} \mathrm{NMR}\left(75 \mathrm{MHz}, \mathrm{CDCl}_{3}\right) \delta: 164.5,156.1$, 151.2, 130.2, 130.1, 129.9, 129.9, 129.8, 129.8, 129.7, 129.7,
$129.6,129.6,129.5,129.5,129.4,129.4,128.7,128.0,115.1$, 111.5, 104.6, 73.6, 59.6, 57.3, 56.3, 35.7, 49.3; ESI-MS, m/z calculated for $\mathrm{C}_{28} \mathrm{H}_{26} \mathrm{ClF}_{2} \mathrm{~N}_{7} \mathrm{O} 549.2$, found $[\mathrm{M}+1]^{+} 550.3$.

Compound Ie: I-(IH-I,2,4-triazol- |-yl)2-(2,4-difluorophenyl)-3-\{N-benzyl- $N$ [( I-(3-chlorobenzyl)-I H-I,2,3-triazol-4yl)methyl]amino\}-2-propanol

MP: $112.0^{\circ} \mathrm{C}-113.8^{\circ} \mathrm{C} ;{ }^{1} \mathrm{H}$ NMR $\left(300 \mathrm{MHz} \mathrm{CDCl}_{3}\right) \delta: 8.07$ $(1 \mathrm{H}, \mathrm{s}$, triazole-H), $7.74(1 \mathrm{H}, \mathrm{s}$, triazole-H), 7.68-7.59 $(1 \mathrm{H}$, $\mathrm{m}$, Ar-H), 7.36-7.10 (8H, m, Ar-H, triazole-H), 6.94-6.70 $(2 \mathrm{H}, \mathrm{m}, \mathrm{Ar}-\mathrm{H}), 5.52$ (2H, s, Ar- $\left.\mathrm{CH}_{2}-\right), 4.54-4.36$ (2H, m, $\left.\mathrm{CH}_{2}\right), 3.69-3.37$ (4H, m, $\left.-\mathrm{CH}_{2}-\mathrm{N}-\mathrm{CH}_{2}-\right), 3.27-2.72(2 \mathrm{H}, \mathrm{m}$, $\left.\mathrm{CH}_{2}\right) ;{ }^{13} \mathrm{C} \mathrm{NMR}\left(75 \mathrm{MHz}, \mathrm{CDCl}_{3}\right) \delta: 164.3,156.2,151.1$, 130.1, 130.0, 129.9, 129.9, 129.9, 129.8, 129.8, 129.7, 129.6, 129.6, 129.5, 129.5, 129.4, 129.3, 128.6, 128.1, 115.3, 111.4, 104.2, 73.4, 59.4, 57.2, 56.2, 35.6, 49.2; ESI-MS, m/z calculated for $\mathrm{C}_{28} \mathrm{H}_{26} \mathrm{ClF}_{2} \mathrm{~N}_{7} \mathrm{O} 549.2$, found $[\mathrm{M}+1]^{+} 550.3$.

Compound If: I-(IH-I,2,4-triazol- I-yl)-2(2,4-difluorophenyl)-3-\{N-benzyl-N[( I-(4-chlorobenzyl)-I H-I,2,3-triazol-4yl)methyl]amino\}-2-propanol

MP: $111.2^{\circ} \mathrm{C}-113.0^{\circ} \mathrm{C} ;{ }^{1} \mathrm{H}$ NMR $\left(300 \mathrm{MHz}, \mathrm{CDCl}_{3}\right) \delta: 8.07$ $(1 \mathrm{H}, \mathrm{s}$, triazole-H), $7.73(1 \mathrm{H}, \mathrm{s}$, triazole-H), 7.68-7.60 $(1 \mathrm{H}$, m, Ar-H), 7.40-7.13 (8H, m, Ar-H, triazole-H), 6.85-6.70 $(2 \mathrm{H}, \mathrm{m}, \mathrm{Ar}-\mathrm{H}), 5.52$ (2H, s, Ar- $\left.\mathrm{CH}_{2}-\right)$, 4.55-4.35 (2H, m, $\mathrm{CH}_{2}$ ), 3.68-3.39 (4H, m, - $\left.\mathrm{CH}_{2}-\mathrm{N}_{-} \mathrm{CH}_{2}-\right), 3.27-2.83$ (2H, m, $\left.\mathrm{CH}_{2}\right) ;{ }^{13} \mathrm{C} \mathrm{NMR}\left(75 \mathrm{MHz}, \mathrm{CDCl}_{3}\right) \delta: 164.5,156.1,151.3$, 130.0, 130.0, 129.9, 129.9, 129.8, 129.8, 129.8, 129.7, 129.7, 129.6, 129.6, 129.5, 129.4, 129.4, 128.5, 128.3, 115.1, 111.7, 104.7, 73.1, 59.7, 57.1, 56.1, 35.7, 49.0; ESI-MS, m/z calculated for $\mathrm{C}_{28} \mathrm{H}_{26} \mathrm{ClF}_{2} \mathrm{~N}_{7} \mathrm{O} 549.2$, found $[\mathrm{M}+1]^{+} 550.4$.

Compound Ig: I-(|H-I,2,4-triazol- I-y|)2-(2,4-difluorophenyl)-3-\{N-benzyl- $N$ [( I-(2-bromobenzyl)-IH-I,2,3-triazol-4yl)methyl]amino\}-2-propanol

MP: $116.8^{\circ} \mathrm{C}-118.2^{\circ} \mathrm{C} ;{ }^{1} \mathrm{H}$ NMR $\left(300 \mathrm{MHz}, \mathrm{CDCl}_{3}\right) \delta: 8.07$ $(1 \mathrm{H}$, s, triazole-H), $7.75(1 \mathrm{H}, \mathrm{s}$, triazole- $\mathrm{H}), 7.63-7.61(1 \mathrm{H}$, m, Ar-H), 7.57-7.51 (2H, m, Ar-H), 7.27-7.11 (8H, m, Ar-H, triazole-H), 6.82-6.70 (2H, m, Ar-H), $5.52\left(2 \mathrm{H}, \mathrm{s}, \mathrm{Ar}-\mathrm{CH}_{2}\right)$, 4.51-4.36 (2H, m, $\left.\mathrm{CH}_{2}\right), 3.73-3.40\left(4 \mathrm{H}, \mathrm{m},-\mathrm{CH}_{2}-\mathrm{N}-\mathrm{CH}_{2}-\right)$, 3.30-2.85 (2H, m, $\left.\mathrm{CH}_{2}\right) ;{ }^{13} \mathrm{C} \mathrm{NMR}\left(75 \mathrm{MHz} \mathrm{CDCl}_{3}\right) \delta$ : 163.5, 159.6, 151.4, 136.2, 134.8, 131.8, 131.8, 131.5, 130.5, 130.4 , 130.4, 130.3, 129.8, 129.8, 129.7, 129.2, 129.2, 128.5, 128.5, 128.3, 112.2, 104.8, 73.4, 62.1, 58.2, 53.2, 52.0, 48.0; 
ESI-MS, $\mathrm{m} / \mathrm{z}$ calculated for $\mathrm{C}_{28} \mathrm{H}_{26} \mathrm{BrF}_{2} \mathrm{~N}_{7} \mathrm{O}$ 593.1, found $[\mathrm{M}+1]^{+}$594.3.

Compound Ih: I-(IH-I,2,4-triazol- I-yl)2-(2,4-difluorophenyl)-3-\{N-benzyl- $N$ [( I-(3-bromobenzyl)-IH-I,2,3-triazol-4yl)methyl]amino\}-2-propanol

MP: $101.1^{\circ} \mathrm{C}-102.1{ }^{\circ} \mathrm{C} ;{ }^{1} \mathrm{H}$ NMR $\left(300 \mathrm{MHz}, \mathrm{CDCl}_{3}\right) \delta: 8.03$ $(1 \mathrm{H}, \mathrm{s}$, triazole- $\mathrm{H}), 7.73(1 \mathrm{H}, \mathrm{s}$, triazole- $\mathrm{H}), 7.64-7.60(1 \mathrm{H}$, m, Ar-H), 7.54-7.51 (2H, m, Ar-H), 7.29-7.14 (8H, m, Ar-H, triazole-H), 6.84-6.70 (2H, m, Ar-H), $5.54\left(2 \mathrm{H}, \mathrm{s}, \mathrm{Ar}_{-} \mathrm{CH}_{2}\right)$, 4.55-4.35 (2H, m, $\left.\mathrm{CH}_{2}\right), 3.74-3.43\left(4 \mathrm{H}, \mathrm{m},-\mathrm{CH}_{2}-\mathrm{N}-\mathrm{CH}_{2}-\right)$, 3.30-2.87 (2H, m, $\left.\mathrm{CH}_{2}\right) ;{ }^{13} \mathrm{C}$ NMR $\left(75 \mathrm{MHz}, \mathrm{CDCl}_{3}\right) \delta$ : 163.2, 158.6, 151.0, 144.6, 133.6, 132.9, 130.7, 129.9, 129.9, $129.8,129.8,129.7,129.7,129.6,129.5,129.5,129.4,128.5$, $128.2,122.7,111.5,104.5,73.8,59.8,57.8,56.2,53.5,49.2$; ESI-MS, $\mathrm{m} / \mathrm{z}$ calculated for $\mathrm{C}_{28} \mathrm{H}_{26} \mathrm{BrF}_{2} \mathrm{~N}_{7} \mathrm{O} 593.1$, found $[\mathrm{M}+1]^{+}$594.5.

Compound Ii: I-(IH-I,2,4-triazol- I-yl)-2(2,4-difluorophenyl)-3-\{N-benzyl-N[( I-(4-bromobenzyl)-IH-I,2,3-triazol-4yl)methyl]amino\}-2-propanol

MP: $108.6^{\circ} \mathrm{C}-111.0^{\circ} \mathrm{C} ;{ }^{1} \mathrm{H}$ NMR $\left(300 \mathrm{MHz}, \mathrm{CDCl}_{3}\right) \delta: 8.16$ $(1 \mathrm{H}, \mathrm{s}$, triazole-H), $7.79(1 \mathrm{H}, \mathrm{s}$, triazole- $\mathrm{H}), 7.64-7.62(1 \mathrm{H}$, m, Ar-H), 7.62-7.59 (2H, m, Ar-H), 7.29-7.16 (8H, m, Ar-H, triazole-H), 6.83-6.70 (2H, m, Ar-H), $5.50\left(2 \mathrm{H}, \mathrm{s}, \mathrm{Ar}_{-} \mathrm{CH}_{2}\right)$, 4.58-4.12 (2H, m, $\left.\mathrm{CH}_{2}\right), 3.70-3.49\left(4 \mathrm{H}, \mathrm{m},-\mathrm{CH}_{2}-\mathrm{N}_{-}-\mathrm{CH}_{2}-\right)$, 3.30-2.91 (2H, m, $\left.\mathrm{CH}_{2}\right) ;{ }^{13} \mathrm{C}$ NMR $\left(75 \mathrm{MHz}, \mathrm{CDCl}_{3}\right) \delta$ : 163.7, 159.5, 151.3, 136.1, 134.7, 131.7, 131.7, 131.3, 130.1, 130.2, 130.1, 130.0, 129.9, 129.9, 129.8, 129.7, 129.6, 128.7, 128.7, 128.3, 112.1, 104.5, 73.3, 62.0, 58.1, 53.1, 52.0, 48.3; ESI-MS, m/z calculated for $\mathrm{C}_{28} \mathrm{H}_{26} \mathrm{BrF}_{2} \mathrm{~N}_{7} \mathrm{O} 593.1$, found $[\mathrm{M}+1]^{+}$594.6.

Compound Ij: I-(IH-I,2,4-triazol- I-yl)-2(2,4-difluorophenyl)-3-\{N-benzyl-N[( I-(2-methylbenzyl)- IH-I,2,3-triazol-4yl)methyl]amino\}-2-propanol

MP: $90.6^{\circ} \mathrm{C}-92.1^{\circ} \mathrm{C}$; ${ }^{1} \mathrm{H}$ NMR $\left(300 \mathrm{MHz}, \mathrm{CDCl}_{3}\right) \delta: 8.08$ $(1 \mathrm{H}, \mathrm{s}$, triazole- $\mathrm{H}), 7.69(1 \mathrm{H}, \mathrm{s}$, triazole- $\mathrm{H}), 7.62-7.57(1 \mathrm{H}$, m, Ar-H), 7.35-7.20 (10H, m, Ar-H, triazole-H), 6.83-6.67 $(2 \mathrm{H}, \mathrm{m}, \mathrm{Ar}-\mathrm{H}), 5.56\left(2 \mathrm{H}, \mathrm{s}, \mathrm{Ar}-\mathrm{CH}_{2}\right), 4.54-4.33(2 \mathrm{H}, \mathrm{m}$, $\left.\mathrm{CH}_{2}\right), 3.72-3.49\left(4 \mathrm{H}, \mathrm{m},-\mathrm{CH}_{2}-\mathrm{N}-\mathrm{CH}_{2}-\right), 3.30-2.91(2 \mathrm{H}, \mathrm{m}$, $\left.\mathrm{CH}_{2}\right), 2.30\left(3 \mathrm{H}, \mathrm{s}, \mathrm{Ar}-\mathrm{CH}_{3}\right) ;{ }^{13} \mathrm{C} \mathrm{NMR}\left(75 \mathrm{MHz}, \mathrm{CDCl}_{3}\right)$

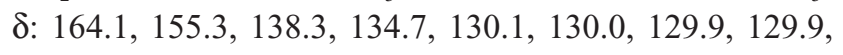
129.8, 129.7, 129.7, 129.6, 129.6, 129.5, 129.4, 129.3, 129.2, 128.7, 128.6, 125.1, 111.8, 104.1, 72.3, 59.7, 57.3, 55.3, 54.0,
49.3, 22.3; ESI-MS, m/z calculated for $\mathrm{C}_{29} \mathrm{H}_{29} \mathrm{~F}_{2} \mathrm{~N}_{7} \mathrm{O} 529.6$, found $[\mathrm{M}+1]^{+} 530.6$.

Compound Ik: I-(|H-I,2,4-triazol- I-yl)2-(2,4-difluorophenyl)-3-\{N-benzyl- $N$ [( I-(3-methylbenzyl)-IH-I,2,3-triazol-4yl)methyl]amino\}-2-propanol

MP: $92.6^{\circ} \mathrm{C}-94.1^{\circ} \mathrm{C} ;{ }^{1} \mathrm{H}$ NMR (300 $\left.\mathrm{MHz}, \mathrm{CDCl}_{3}\right)$ $\delta: 8.07$ $(1 \mathrm{H}, \mathrm{s}$, triazole- $\mathrm{H}), 7.78(1 \mathrm{H}, \mathrm{s}$, triazole- $\mathrm{H}), 7.70-7.58(1 \mathrm{H}$, $\mathrm{m}, \mathrm{Ar}-\mathrm{H}), 7.32-7.08$ (10H, m, Ar-H, triazole-H), 6.83-6.69 $(2 \mathrm{H}, \mathrm{m}, \mathrm{Ar}-\mathrm{H}), 5.56\left(2 \mathrm{H}, \mathrm{s}, \mathrm{Ar}-\mathrm{CH}_{2}\right), 4.69-4.35(2 \mathrm{H}, \mathrm{m}$, $\left.\mathrm{CH}_{2}\right), 3.75-3.45\left(4 \mathrm{H}, \mathrm{m},-\mathrm{CH}_{2}-\mathrm{N}-\mathrm{CH}_{2}-\right), 3.30-2.90(2 \mathrm{H}, \mathrm{m}$, $\left.\mathrm{CH}_{2}\right), 2.37$ (3H, s, $\left.\mathrm{Ar}-\mathrm{CH}_{3}\right) ;{ }^{13} \mathrm{C} \mathrm{NMR}\left(75 \mathrm{MHz}, \mathrm{CDCl}_{3}\right) \delta$ : 163.5, 155.1, 138.1, 134.6, 130.0, 129.9, 129.9, 129.8, 129.8, $129.7,129.7,129.6,129.6,129.5,129.5,129.4,129.2,128.8$, 128.6, 125.3, 111.7, 104.3, 72.5, 59.8, 57.5, 55.8, 54.1, 49.8, 22.5; ESI-MS, $\mathrm{m} / \mathrm{z}$ calculated for $\mathrm{C}_{29} \mathrm{H}_{29} \mathrm{~F}_{2} \mathrm{~N}_{7} \mathrm{O} 529.6$, found $[\mathrm{M}+1]^{+} 530.5$.

Compound II: I-(IH-I,2,4-triazol- I-yl)-2(2,4-difluorophenyl)-3-\{N-benzyl-N[( I-(4-methylbenzyl)-I H-I,2,3-triazol-4yl)methyl]amino\}-2-propanol

MP: $91.2^{\circ} \mathrm{C}-93.0^{\circ} \mathrm{C} ;{ }^{1} \mathrm{H}$ NMR $\left(300 \mathrm{MHz}, \mathrm{CDCl}_{3}\right) \delta: 8.06$ $(1 \mathrm{H}, \mathrm{s}$, triazole-H), $7.71(1 \mathrm{H}, \mathrm{s}$, triazole- $\mathrm{H}), 7.66-7.57(1 \mathrm{H}$, $\mathrm{m}, \mathrm{Ar}-\mathrm{H}), 7.29-6.97$ (10H, m, Ar-H, triazole-H), 6.82-6.67 $(2 \mathrm{H}, \mathrm{m}, \mathrm{Ar}-\mathrm{H}), 5.50\left(2 \mathrm{H}, \mathrm{s}, \mathrm{Ar}-\mathrm{CH}_{2}\right), 4.51-4.35(2 \mathrm{H}, \mathrm{m}$, $\left.\mathrm{CH}_{2}\right), 3.72-3.45\left(4 \mathrm{H}, \mathrm{m},-\mathrm{CH}_{2}-\mathrm{N}-\mathrm{CH}_{2}-\right), 3.33-2.90(2 \mathrm{H}, \mathrm{m}$, $\left.\mathrm{CH}_{2}\right), 2.38\left(3 \mathrm{H}, \mathrm{s}, \mathrm{Ar}-\mathrm{CH}_{3}\right) ;{ }^{13} \mathrm{C} \mathrm{NMR}\left(75 \mathrm{MHz}, \mathrm{CDCl}_{3}\right) \delta$ : 163.9, 155.7, 138.2, 134.5, 130.0, 130.0, 129.9, 129.9, 129.8, $129.8,129.7,129.7,129.6,129.6,129.5,129.3,129.3,128.5$, 128.3, 125.3, 111.7, 104.5, 72.6, 59.5, 57.1, 55.1, 54.1, 49.7, 22.8; ESI-MS, m/z calculated for $\mathrm{C}_{29} \mathrm{H}_{29} \mathrm{~F}_{2} \mathrm{~N}_{7} \mathrm{O} 529.6$, found $[\mathrm{M}+1]^{+} 530.7$.

Compound Im: I-(|H-I,2,4-triazol- I-yl)2-(2,4-difluorophenyl)-3-\{N-benzyl- $N$ [( I-(2-cyanobenzyl)- IH- I,2,3-triazol-4-yl) methyl]amino\}-2-propanol

MP: $79.6^{\circ} \mathrm{C}-81.4^{\circ} \mathrm{C}$; ${ }^{1} \mathrm{H}$ NMR $\left(300 \mathrm{MHz}, \mathrm{CDCl}_{3}\right) \delta: 8.07$ $(1 \mathrm{H}, \mathrm{s}$, triazole- $\mathrm{H}), 7.76(1 \mathrm{H}, \mathrm{s}$, triazole- $\mathrm{H}), 7.65-7.63(1 \mathrm{H}$, $\mathrm{m}, \mathrm{Ar}-\mathrm{H}), 7.54-7.21$ (10H, m, Ar-H, triazole-H), 6.85-6.71 $(2 \mathrm{H}, \mathrm{m}, \mathrm{Ar}-\mathrm{H}), 5.76\left(2 \mathrm{H}, \mathrm{s}, \mathrm{Ar}-\mathrm{CH}_{2}\right), 4.58-4.38(2 \mathrm{H}, \mathrm{m}$, $\left.\mathrm{CH}_{2}\right), 3.75-3.43\left(4 \mathrm{H}, \mathrm{m},-\mathrm{CH}_{2}-\mathrm{N}-\mathrm{CH}_{2}-\right), 3.30-2.87(2 \mathrm{H}, \mathrm{m}$, $\left.\mathrm{CH}_{2}\right), 2.38\left(3 \mathrm{H}, \mathrm{s}, \mathrm{Ar}-\mathrm{CH}_{3}\right) ;{ }^{13} \mathrm{C} \mathrm{NMR}\left(75 \mathrm{MHz}, \mathrm{CDCl}_{3}\right) \delta$ : 163.7, 158.7, 152.1, 142.2, 133.1, 131.2, 130.3, 130.1, 129.9, $129.9,129.8,129.8,129.7,129.6,129.5,128.9,128.5,127.5$, $126.9,118.2,113.5,111.7,104.7,73.5,61.3,58.1,56.2$, 
53.1, 50.1; ESI-MS, m/z calculated for $\mathrm{C}_{29} \mathrm{H}_{26} \mathrm{~F}_{2} \mathrm{~N}_{8} \mathrm{O} 540.2$, found $[\mathrm{M}+1]^{+}$541.3.

Compound In: I-(IH-I,2,4-triazol- I-yl)2-(2,4-difluorophenyl)-3-\{N-benzyl-N[( I-(3-cyanobenzyl)- IH-I,2,3-triazol-4-yl) methyl]amino\}-2-propanol

MP: $82.2^{\circ} \mathrm{C}-83.5^{\circ} \mathrm{C} ;{ }^{1} \mathrm{H} \mathrm{NMR}\left(300 \mathrm{MHz}, \mathrm{CDCl}_{3}\right) \delta: 8.02$ $(1 \mathrm{H}, \mathrm{s}$, triazole-H), $7.75(1 \mathrm{H}, \mathrm{s}$, triazole-H), 7.66-7.63 $(1 \mathrm{H}$, $\mathrm{m}$, Ar-H), 7.53-7.15 (10H, m, Ar-H, triazole-H), 6.85-6.72 $(2 \mathrm{H}, \mathrm{m}, \mathrm{Ar}-\mathrm{H}), 5.59\left(2 \mathrm{H}, \mathrm{s}, \mathrm{Ar}-\mathrm{CH}_{2}\right), 4.58-4.35(2 \mathrm{H}, \mathrm{m}$, $\left.\mathrm{CH}_{2}\right), 3.73-3.41$ (4H, m, $\left.-\mathrm{CH}_{2}-\mathrm{N}-\mathrm{CH}_{2}-\right), 3.30-2.82(2 \mathrm{H}, \mathrm{m}$, $\mathrm{CH}_{2}$ ); ${ }^{13} \mathrm{C} \mathrm{NMR}\left(75 \mathrm{MHz}, \mathrm{CDCl}_{3}\right) \delta: 163.3,158.5,152.3$, $142.3,133.2,131.3,130.1,130.0,129.9,129.9,129.8,129.7$, 129.6, 129.3, 128.9, 128.7, 127.5, 127.4, 126.3, 118.4, 113.3, $112.0,104.5,73.7,61.5,58.3,56.3,53.6,50.2 ;$ ESI-MS, m/z calculated for $\mathrm{C}_{29} \mathrm{H}_{26} \mathrm{~F}_{2} \mathrm{~N}_{8} \mathrm{O} 540.2$, found $[\mathrm{M}+1]^{+} 541.4$.

Compound Io: I-(|H-I,2,4-triazol- I-yl)2-(2,4-difluorophenyl)-3-\{N-benzyl-N[( I-(4-cyanobenzyl)- IH-I,2,3-triazol-4-yl) methyl]amino\}-2-propanol

MP: $78.8^{\circ} \mathrm{C}-80.4^{\circ} \mathrm{C} ;{ }^{1} \mathrm{H}$ NMR $\left(300 \mathrm{MHz}, \mathrm{CDCl}_{3}\right) \delta: 8.08$ $(1 \mathrm{H}, \mathrm{s}$, triazole-H), $7.75(1 \mathrm{H}, \mathrm{s}$, triazole-H), 7.71-7.63 $(1 \mathrm{H}$, m, Ar-H), 7.37-7.22 (10H, m, Ar-H, triazole-H), 6.86-6.72 $(2 \mathrm{H}, \mathrm{m}, \mathrm{Ar}-\mathrm{H}), 5.62\left(2 \mathrm{H}, \mathrm{s}, \mathrm{Ar}-\mathrm{CH}_{2}\right), 4.62-4.34(2 \mathrm{H}, \mathrm{m}$, $\left.\mathrm{CH}_{2}\right), 3.74-3.42\left(4 \mathrm{H}, \mathrm{m},-\mathrm{CH}_{2}-\mathrm{N}-\mathrm{CH}_{2}-\right), 3.30-2.86(2 \mathrm{H}, \mathrm{m}$, $\left.\mathrm{CH}_{2}\right) ;{ }^{13} \mathrm{C} \mathrm{NMR}\left(75 \mathrm{MHz}, \mathrm{CDCl}_{3}\right) \delta: 163.2,158.7,152.1$, $142.1,133.1,131.1,130.0,130.0,129.9,129.8,129.8,129.7$, $129.5,129.0,128.7,128.7,127.4,127.3,126.5,118.0,113.1$, 112.1, 104.9, 73.1, 61.2, 58.1, 56.1, 53.8, 50.0; ESI-MS, m/z calculated for $\mathrm{C}_{29} \mathrm{H}_{26} \mathrm{~F}_{2} \mathrm{~N}_{8} \mathrm{O} 540.2$, found $[\mathrm{M}+1]^{+} 541.5$.

Compound Ip: I-(|H-I,2,4-triazol- |-yl)2-(2,4-difluorophenyl)-3-\{N-benzyl-N[(I-(2-nitrobenzyl)-IH-I,2,3-triazol-4-yl) methyl]amino\}-2-propanol

MP: $80.2^{\circ} \mathrm{C}-81.5^{\circ} \mathrm{C}$; ${ }^{1} \mathrm{H}$ NMR $\left(300 \mathrm{MHz}, \mathrm{CDCl}_{3}\right) \delta$ : 8.17-8.15 (1H, m, Ar-H), 8.06 (1H, s, triazole-H), 7.72
(1H, s, triazole-H), 7.65-7.59 (3H, m, Ar-H), 7.29-7.17 $(7 \mathrm{H}, \mathrm{m}, \mathrm{Ar}-\mathrm{H}$, triazole-H), 6.83-6.72 (2H, m, Ar-H), 5.93 $\left(2 \mathrm{H}, \mathrm{s}, \mathrm{Ar}-\mathrm{CH}_{2}\right), 4.58-4.38\left(2 \mathrm{H}, \mathrm{m}, \mathrm{CH}_{2}\right), 3.76-3.41(4 \mathrm{H}, \mathrm{m}$, $\left.-\mathrm{CH}_{2}-\mathrm{N}-\mathrm{CH}_{2}-\right), 3.30-2.97\left(2 \mathrm{H}, \mathrm{m}, \mathrm{CH}_{2}\right) ;{ }^{13} \mathrm{C} \mathrm{NMR}(75 \mathrm{MHz}$, $\left.\mathrm{CDCl}_{3}\right)$ \&: $163.5,158.7,148.3,136.5,133.7,130.1,130.0$, $129.9,129.9,129.8,129.8,129.7,129.6,129.6,129.5,129.5$, 129.4, 128.7, 123.8, 122.5, 111.2, 104.7, 73.6, 59.1, 57.6, 55.3, 53.1, 49.0; ESI-MS, m/z calculated for $\mathrm{C}_{28} \mathrm{H}_{26} \mathrm{~F}_{2} \mathrm{~N}_{8} \mathrm{O}_{3}$ 560.2 , found $[\mathrm{M}+1]^{+} 561.4$.

Compound Iq: I-( $\mid \mathrm{H}-\mathrm{I}, 2,4-$ triazol- I-yl)2-(2,4-difluorophenyl)-3- $\{N$-benzyl- $N$ [( I-(3-nitrobenzyl)-IH-I,2,3-triazol-4-yl) methyl]amino\}-2-propanol

MP: $82.0^{\circ} \mathrm{C}-83.6^{\circ} \mathrm{C} ;{ }^{1} \mathrm{H}$ NMR $\left(300 \mathrm{MHz}, \mathrm{CDCl}_{3}\right) \delta: 8.04$ $(1 \mathrm{H}, \mathrm{s}$, triazole-H), $7.73(1 \mathrm{H}, \mathrm{s}$, triazole-H), 7.63-7.60 $(1 \mathrm{H}$, m, Ar-H), 7.50-7.17 (10H, m, Ar-H, triazole-H), 6.84-6.70 (2H, m, Ar-H), $5.50\left(2 \mathrm{H}, \mathrm{s}, \mathrm{Ar}-\mathrm{CH}_{2}\right), 4.56-4.35\left(2 \mathrm{H}, \mathrm{m}, \mathrm{CH}_{2}\right)$, 3.71-3.41 (4H, m, $\left.-\mathrm{CH}_{2}-\mathrm{N}-\mathrm{CH}_{2}-\right)$, 3.32-2.90 (2H, m, $\left.\mathrm{CH}_{2}\right)$; ${ }^{13} \mathrm{C} \mathrm{NMR}\left(75 \mathrm{MHz}, \mathrm{CDCl}_{3}\right) \delta: 162.5,154.3,136.7,132.0$, 131.1, 130.3, 129.9, 129.9, 129.8, 129.8, 129.7, 129.6, 129.6, $129.5,129.4,129.0,128.6,127.6,126.6,123.2,111.7,104.2$, 73.3, 59.8, 57.6, 56.2, 53.4, 49.2; ESI-MS, m/z calculated for $\mathrm{C}_{28} \mathrm{H}_{26} \mathrm{~F}_{2} \mathrm{~N}_{8} \mathrm{O}_{3} 560.2$, found $[\mathrm{M}+1]^{+}$561.3.

Compound Ir: I-(IH-I,2,4-triazol- I-yl)2-(2,4-difluorophenyl)-3-\{N-benzyl- $N$ [( I-(4-nitrobenzyl)-I H-I,2,3-triazol-4-yl) methyl]amino\}-2-propanol

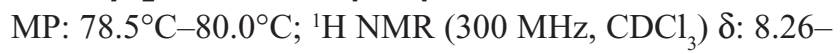
$8.15(2 \mathrm{H}, \mathrm{m}, \mathrm{Ar}-\mathrm{H}), 8.05(1 \mathrm{H}, \mathrm{s}$, triazole-H), $7.74(1 \mathrm{H}, \mathrm{s}$, triazole-H), 7.64-7.20 (9H, m, Ar-H, triazole-H), 6.85-6.71 (2H, m, Ar-H), 5.67 (2H, s, Ar- $\left.\mathrm{CH}_{2}\right), 4.60-4.34\left(2 \mathrm{H}, \mathrm{m}, \mathrm{CH}_{2}\right)$, 3.72-3.42 (4H, m, - $\mathrm{CH}_{2}-\mathrm{N}-\mathrm{CH}_{2}-$ ), 3.36-2.91 (2H, m, $\left.\mathrm{CH}_{2}\right)$; ${ }^{13} \mathrm{C}$ NMR (75 MHz, $\mathrm{CDCl}_{3}$ ) $\delta: 162.7,158.5,148.6,136.7$, $133.9,130.4,130.0,129.9,129.8,129.8,129.7,129.7,129.6$, $129.6,129.5,129.4,129.4,128.6,123.8,122.7,111.8,104.4$, 73.2, 59.8, 57.5, 55.8, 53.2, 49.2; ESI-MS, m/z calculated for $\mathrm{C}_{28} \mathrm{H}_{26} \mathrm{~F}_{2} \mathrm{~N}_{8} \mathrm{O}_{3} 560.2$, found $[\mathrm{M}+1]^{+} 561.5$.
Drug Design, Development and Therapy

\section{Publish your work in this journal}

Drug Design, Development and Therapy is an international, peerreviewed open-access journal that spans the spectrum of drug design and development through to clinical applications. Clinical outcomes, patient safety, and programs for the development and effective, safe, and sustained use of medicines are a feature of the journal, which

\section{Dovepress}

has also been accepted for indexing on PubMed Central. The manuscript management system is completely online and includes a very quick and fair peer-review system, which is all easy to use. Visit http://www.dovepress.com/testimonials.php to read real quotes from published authors.

Submit your manuscript here: http://www.dovepress.com/drug-design-development-and-therapy-journal 